\title{
Therapeutic Phytochemical Actives for Potential Control of SARS-CoV-2
}

\author{
Dipjyoti Dey', Nirban Dey1, Shalini Ghosh¹, Imran Khan², Padma Thiagarajan ${ }^{1, *}$ \\ ${ }^{1}$ Department of Biomedical Sciences, School of Biosciences and Technology, Vellore Institute of Technology, Vellore, Tamil Nadu, \\ INDIA. \\ ${ }^{2}$ Department of Molecular Biology, Bezmialem Vakif University, Istanbul, TURKEY.
}

\begin{abstract}
Background: Drug development strategies for treating COVID-19 focus on actives that either physically block angiotensin-converting enzyme-2 (ACE-2) receptors (viral entry point), or those, which inactivate viral proteases like 3CLpro or RdRp, inside the infected host cells. Objectives: The objective of the present study is to virtually screen phytochemicals for both these purposes. Methods: Molecular docking, molecular dynamic simulation (MDS) and multiple sequence alignment were employed. Results: All the screened phytochemical actives showed negative binding energies with their respective targets, attesting good complex stabilities. Among each set of ten actives, for blocking ACE-2 receptors and for inactivation of 3CLpro and RdRp, DichamanetinACE-2, Glabrene-3CLpro and Naringenin-RdRp complexes were most stable, with binding energies of $-9.8,-9.11$ and $-7.7 \mathrm{Kcal} / \mathrm{mol}$ respectively. MDS studies of these representative actives and their complexes, also attested to complex stabilities. Multiple sequence alignment analysis of nine significant amino acid residues of the Homo sapiens ACE-2 receptor, with nine different species, showed conservation of several residues. Conclusion: A set of phytochemicals actives can block ACE-2 receptors and prevent the entry of SARS-CoV-2 into host endothelial cells. Two other sets of actives can inactivate viral 3CLpro and RdRp enzymes and prevent replication of SARS-CoV-2 inside host cells. They all can hence be further explored for the control of COVID-19.
\end{abstract}

Key words: COVID-19, SARS-CoV-2, Angiotensin Converting Enzyme-2, 3CLpro, RdRp.

\section{INTRODUCTION}

The global medical community is racing to find therapeutic solutions for the contagious novel coronavirus disease (COVID-19), transmitted by SARS-CoV-2. The disease leads to respiratory distress and organ failure, primarily in immune-compromised and comorbid patients. ${ }^{1-3}$ To date, no synthetic or natural compounds have been medically validated for its treatment. The quest for effective vaccines is also on-going as such products have to pass through several phases of clinical trials before they are released commercially.

World-wide, the development of therapeutic actives or drugs that can influence key and parallel pathways for COVID control is hence an area of intense research. The structural conformations and functional groups of such drug candidates can contribute to fortify and protect host cells at different levels. Drugs may block the host endothelial cell surface receptors to which the SARS-CoV-2's spike protein binds, for facilitating an entry. Alternatively, drug molecules can also deactivate vital viral proteases inside infected cells. When employed individually or in tandem, both strategies aim to reduce viral loads and prevent aggravation of symptoms, thus leading to successful intervention. Angiotensin-Converting Enzyme 2 (ACE2) protein receptors are the main entry points for the invasion of SARS-CoV-2 into the host cells, although other entry points may also exist. ${ }^{4-6}$ After entry, an eleven-time proteolytic cleavage of a single transcribed
Submission Date: 29-07-2020; Revision Date: 02-12-2020; Accepted Date: 31-03-2021

DOI: 10.5530/ijper.55.2.90 Correspondence: Dr. Padma Thiagarajan Professor (Higher Academic Grade), School of Biosciences and Technology, Vellore Institute of Technology, Vellore-632014, Tamil Nadu, INDIA.

Phone no: +919500359485 Email id: p.padma@vit.ac.in

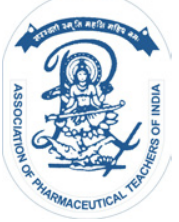

www.ijper.org 
viral poly-protein, by viral 3CLpro, yields mature functional proteins. ${ }^{7-9}$ A viral non-structural protein nsp12, also known as RNA dependent RNA polymerase (RdRp), further catalyses the synthesis of viral RNA, by using nsp7 and nsp8 as cofactors. RdRp thus assumes a crucial role in the replication and transcription cycle of SARS-CoV-2. ${ }^{10,11}$

In the present study, virtual interactions of several phytochemical actives, with ACE-2 receptor protein (for blocking viral entry into target cells), 3CLpro (for inactivating it and thus preventing poly-protein cleavage) and RdRp (for blocking viral RNA synthesis), have been studied. Since phytochemicals are abundant in the plant kingdom as constituents of natural food products, the need for their multi-step laboratory synthesis is abolished. By serving as instant actives from wholesome dietary sources, they may be therapeutically consumed, for potential infection control. ${ }^{12-14}$

\section{MATERIALS AND METHODS \\ Databases Used}

Information on three protein targets, $v i$; , the ACE2protein receptor, 3CLpro and RdRp (PDB IDs 1R42, ${ }^{15}$ $6 \mathrm{LU} 7,{ }^{16} 6 \mathrm{M} 71^{17}$ respectively) was collected from PDB database. Information on thirty phytochemicals used as ligands, were collected from PubChem. Open Babel ${ }^{18}$ was used to convert their SDF file formats, downloaded from PubChem, to PDB file format. To determine their drug likeliness (Lipinski's Rule of Five) and bioavailabilities, the Swiss ADME $^{19}$ web tool was used.

\section{Molecular Docking And Dynamic Simulation}

Interactions between target proteins and their respective phytochemical ligands were determined by AutoDock version 4.2. Target proteins were first prepared with the ligands by the following necessary steps. ${ }^{20}$ Their active sites were then fixed with specific residues. The respective ligands were positioned to binding sites by using an auto grid and by determining the $\mathrm{x}, \mathrm{y}, \mathrm{z}$ coordinates. AutoDock was carried out for docking each ligand with the individual target protein by making use of Lamarckian genetic algorithm. The number of hydrogen bonds and also binding energies were noted. Hydrophobic interactions and $\pi-\pi$ interactions were also recorded by AutoDock 4.2, PyMol 2.3.2 and Protein-Ligand interaction profiler. ${ }^{21}$ Gromacs $2018^{22}$ was used to carry out this study, till 200ps. Simulation was carried out for the unbound target proteins and the selected target protein-ligand complexes. The topology of the target proteins was generated with the Gromacs 96 53a6 force field. ${ }^{23}$ PRODRG server was used to create the ligand topology files. ${ }^{24}$ Solvation was performed using simple point charge water molecules. Neutralization of the free target proteins and protein-ligand complexes was achieved with counter-ions like $\mathrm{Na}^{+}$and $\mathrm{Cl}^{-}$. After necessary optimizations, simulations were carried out till 200ps. The Gromacs utility files contained data on Root Mean Square Deviations (RMSD) and Root Mean Square Fluctuations (RMSF). ${ }^{25}$

\section{Multiple Sequence Alignment}

ClustalOmega ${ }^{26}$ was used to perform MSA of the target Homo sapiens ACE- 2 receptor protein with that of nine different species.

\section{RESULTS AND DISCUSSION}

Structures of three target proteins, vir., ACE-2 protein receptor, 3CLpro and RdRp (PDB IDs 1R42, 6LU7, 6M71 respectively), were obtained from the Protein Data Bank. ACE-2 protein receptor was chosen since the Receptor Binding Domain (RBD) of the viral spike protein interacts with it to enter into the host's endothelial cells. ${ }^{27}$ 3CLpro (nsp5), contains domains one (residues 8-101), two (residues 102-184) and three, which are involved in the maturation of viral non-structural proteins essential for viral life cycle including replication and transcription. RdRp plays a vital role in the viral replication/transcription complex and it is conserved in all coronavirus species. ${ }^{28}$ Ten phytochemicals, available from plants that can serve as dietary sources, were selected as potential compounds for studies with each protein target, vir., ACE-2 protein receptors, 3CLpro and RdRp [Table 1(a-c)]. Based on their bioavailability and drug-likeliness accordingly to Lipinski's Rule of Five, ${ }^{29}$ they were screened virtually. Lipinski's rule defines the important molecular properties that concern an active's pharmacokinetics in the human body and includes its absorption, distribution, metabolism and excretion. It is vital for drug development wherein a pharmacologically dynamic lead structure is optimized step-wise, for increased activity and selectivity, along with drug-like properties. As per this rule, in general, an orally active drug should have no more than one violation of the following criteria: (a) A molecular weight of less than $500 \mathrm{Da}$ (b) An octanol-water partition coefficient $(\log P$ ) of less than 5 (c) Not more than five hydrogen bond donors (nitrogen or oxygen atoms with one or more hydrogen atoms) (d) Not more than ten hydrogen bond acceptors (nitrogen or oxygen atoms). Except for Baicalin, which showed two violations, all phytochemicals in this study had a bioavailability of 0.5 and obeyed Lipinski's Rule of Five [Table 1(a-c)]. 
Table 1a: Drug-likeliness analysis of phytochemicals targeting ACE2.

\begin{tabular}{|c|c|c|c|c|c|}
\hline $\begin{array}{l}\text { Compound/ } \\
\text { PubChem CID }\end{array}$ & Source & $\begin{array}{c}\text { Molecular } \\
\text { weight } \\
(<500 \mathrm{Da})\end{array}$ & $\log p(<5)$ & $\begin{array}{c}\text { H-Bond } \\
\text { donor }(<5)\end{array}$ & $\begin{array}{l}\text { H-bond } \\
\text { acceptor } \\
(<10)\end{array}$ \\
\hline Dichamanetin/181193 & $\begin{array}{c}\text { Piper sarmetosum (Wild betel) } \\
\text { Polyalthia longifolia } \\
\text { (Ashoka tree, debdaru) }\end{array}$ & 468.50 & 2.79 & 4 & 6 \\
\hline Hetidine/101685340 & $\begin{array}{c}\text { Aconitum heterophyllum } \\
\text { (Indian atees, ativisha) }\end{array}$ & 357.44 & 1.39 & 2 & 5 \\
\hline 6-DeacetyINimbin/10505484 & Azadirachta indica (Neem) & 498.56 & 1.69 & 1 & 8 \\
\hline Lycorine/72378 & Lycoris radiate (Red spider lily) & 287.31 & 1.08 & 2 & 5 \\
\hline Epicatechin gallate/107905 & Camellia sinensis (Green tea) & 442.37 & 0.05 & $7^{*}$ & 10 \\
\hline PhaitanthrinD/ 24970703 & Isatis indigotica (Indigowoad) & 292.29 & 2.07 & 1 & 3 \\
\hline Belladine/441586 & Aconitum heterophyllum (Indian Ativisha ) & 315.41 & 2.70 & 0 & 4 \\
\hline Quercetin/5280343 & Allium cepa (Onion) & 302.24 & -0.56 & 5 & 7 \\
\hline Scutellarein/5281697 & Houttuynia cordata (Chameleon plant) & 286.24 & -0.03 & 4 & 6 \\
\hline Baicalin/64982 & Scutellaria baicalensis (Baikal skullcap) & 446.36 & -1.63 & $6^{*}$ & $11^{*}$ \\
\hline
\end{tabular}

*Represents deviation from Lipinski's rule

\begin{tabular}{|c|c|c|c|c|c|}
\hline $\begin{array}{l}\text { Compound/ } \\
\text { PubChemCID }\end{array}$ & Source & $\begin{array}{c}\text { Molecular } \\
\text { weight }(<500 \\
\mathrm{Da})\end{array}$ & $\begin{array}{c}\text { Logp } \\
(<5)\end{array}$ & $\begin{array}{c}\text { H-Bond } \\
\text { donor } \\
(<5)\end{array}$ & $\begin{array}{l}\text { H-bond } \\
\text { acceptor } \\
(<10)\end{array}$ \\
\hline Glabrene/480774 & $\begin{array}{l}\text { Salvia officinalis (Garden Sage) } \\
\text { Glycyrrhiza glabra licorice (mulethi) }\end{array}$ & 322.35 & 2.65 & 2 & 4 \\
\hline Luteolin/5280445 & $\begin{array}{c}\text { Brassica oleracea var. italica (Broccoli) } \\
\text { Capsicum annum (Chilli pepper) }\end{array}$ & 286.24 & -0.03 & 4 & 6 \\
\hline Ferulic acid/445858 & Salvia officinalis (Garden sage) & 194.18 & 1 & 2 & 4 \\
\hline Epicatechin/72276 & Camellia sinensis (Green tea) & 290.27 & 0.24 & 5 & 6 \\
\hline Apigenin/5280443 & Salvia officinalis (Garden sage) & 270.24 & 0.52 & 3 & 5 \\
\hline Kaempferol/5280863 & $\begin{array}{c}\text { Moringa oleifera (Drumstick tree) } \\
\text { Spinacia oleracea (Spinach) } \\
\text { Brassica oleracea (Cabbage) }\end{array}$ & 286.24 & -0.03 & 4 & 6 \\
\hline Rosmarinic acid /5281792 & Salvia officinalis (Garden sage) & 360.31 & 0.90 & 5 & 8 \\
\hline Caffeic acid/689043 & Salvia officinalis (Garden sage) & 180.16 & 0.70 & 3 & 4 \\
\hline Gallic Acid/ 370 & $\begin{array}{c}\text { Anacardium occidentale (Cashew nut) } \\
\text { Vitis vinifera(Grapes) }\end{array}$ & 170.12 & -0.16 & 4 & 5 \\
\hline Vanillic acid /8468 & Salvia officinalis (Garden sage) & 168.15 & 0.74 & 2 & 4 \\
\hline
\end{tabular}

\begin{tabular}{|c|c|c|c|c|c|}
\hline \multirow[b]{2}{*}{$\begin{array}{c}\text { Compound/PubChem } \\
\text { CID }\end{array}$} & \multirow[b]{2}{*}{ Source } & \multicolumn{4}{|c|}{ Properties } \\
\hline & & $\begin{array}{l}\text { Molecular } \\
\text { weight } \\
\text { (<500 Da) }\end{array}$ & $\operatorname{Logp}(<5)$ & $\begin{array}{c}\text { H-Bond } \\
\text { donor }(<5)\end{array}$ & $\begin{array}{l}\text { H-bond } \\
\text { acceptor } \\
(<10)\end{array}$ \\
\hline Naringenin/932 & $\begin{array}{c}\text { Citrus sinensis (Orange) } \\
\text { Lycopersicon esculentum (Tomato) }\end{array}$ & 272.2 & 0.71 & 3 & 5 \\
\hline Licoflavonol/ 5481964 & Glycyrrhiza uralensis (Chinese liqourice) & 354.35 & 1.09 & 4 & 6 \\
\hline Bicalein/5281605 & $\begin{array}{l}\text { Scutellaria baicalensis (Baikal skullcap) } \\
\text { Scutellaria lateriflora (Blue skullcap ) }\end{array}$ & 270.24 & 0.52 & 3 & 5 \\
\hline Vitexin /5280441 & Crataegus oxycantha (Hawthorn) & 432.38 & -2.02 & 7 & 10 \\
\hline LicocoumarinA/5324358 & Glycyrrhiza glabra (Licorice) & 406.47 & 3.45 & 3 & 5 \\
\hline Liquiritin /503737 & Glycyrrhiza glabra (Licorice) & 418.39 & -0.92 & 5 & 9 \\
\hline Biochanin A /5280373 & Trifolium pratense (Red clover) & 284.26 & 0.77 & 2 & 5 \\
\hline Ellagic acid /5281855 & Fragaria anannassa (Strawberry) & 302.19 & 0.14 & 4 & 8 \\
\hline Licocoumarone /503731 & Glycyrrhiza uralensis (Chinese liqourice) & 340.37 & 2.01 & 3 & 5 \\
\hline Curcumin/969516 & Curcuma longa (Turmeric) & 368.38 & 1.47 & 2 & 6 \\
\hline
\end{tabular}


MDA was performed with the phytochemicals (as ligands) and their respective protein targets, using AutoDock 4.2 for prediction of their binding affinities in terms of the binding energies of their complexes [Table 2(a-c)]. Higher binding affinities of phytochemical ligands to the target proteins are reflected as lower binding energies of the complexes. In this study, binding energies were negative for all complexes, showing a good association of phytochemical ligands and their respective target proteins. When two or more compounds have similar binding energies, the number of hydrogen bonds determines their respective stabilities. These bonds, along with hydrophobic interactions, salt bridges, $\pi$ stacking and cation- $\pi$ interactions, were also analysed using a protein-ligand interaction profiler [Supplementary Table (S1-S3)]. Dichamanetin, Glabrene and Naringenin, with binding energies of $-9.8,-9.11$ and $-7.7 \mathrm{Kcal} / \mathrm{mol}$, showed best affinities for binding with ACE-2 protein receptor, 3CLpro and RdRp respectively. Visualization of their protein-ligand complex structures was done by using Pymol 2.3.2 [Figure 1(a-c)].

Dichamanetin, Glabrene and Naringenin (with no violation of Lipinski's rule) had the least binding energies [Table 2(a-c)] with their respective target proteins. Hence MDS was performed for their target proteins

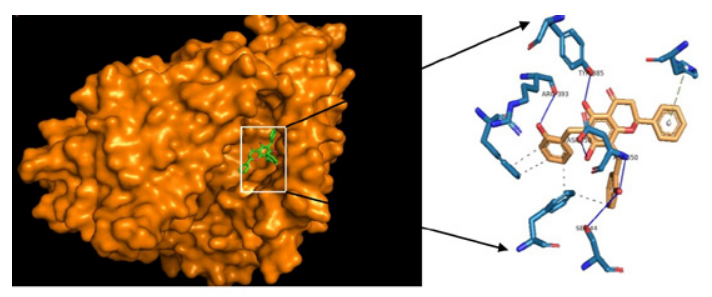

Fig 1a
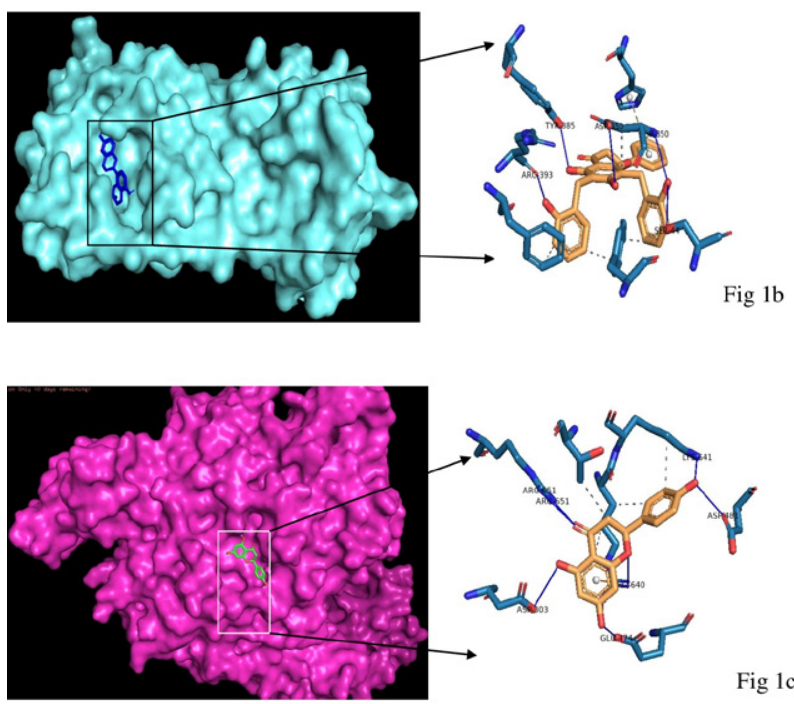

Figure 1: Molecular Docking of ACE-2 with Dichamanetin, 3CLpro with Glabrene and RdRp with Naringenin, along with their bond interactions. and complexes. Root Mean Square Deviation (RMSD) values for each target protein before (blue) and after (red) binding with the respective phytochemical active ligand, as a function of time, are shown in Figure 2(a-c). Compared to free forms of all three target proteins, the bound forms with their respective actives had favourable configurations that impart stability to these complexes and facilitate their formations.

Additional evidence for the stable nature of these protein complexes, when compared to their free forms, is obtained by the Root Mean Square Fluctuation (RMSF) analysis, which shows the flexibility of amino acid residues of target proteins before and after binding to phytochemical ligands to form their respective complexes. Higher RMSF values signify higher flexibilities and lower stabilities and vice versa. RMSF values of single amino acid residues of the target proteins, before (blue) and after (red) binding with the respective phytochemical ligands, are shown in Figure 3(a-c). RMSF values for the active site amino acid residues of ACE- 2 protein receptor before and after binding to Dichamanetin, are given in brackets as follows:

Glu145(0.1105,0.0980), Cys344(0.1181,0.0810), His345(0.1265,0.0880),

Cys361(0.1101,0.0750),His374(0.1198,0.0766), Glu375(0.1155,0.0787),

His 378(0.1455,0.0783), Glu402(0.1988,0.0841), His505(0.1160,0.1026),

Arg514(0.1249,0.1023), Tyr515(0.1318,0.1119), Asn149(0.1323,0.1029),

Arg273(0.0949,0.0727), Pro346(0.1370,0.1057), Thr347(0.1449,0.1194),

Met360(0.1112,0.0742), Lys363(0.1058,0.0849), Thr371(0.1169.0.0776),

Tyr510(0.1194,0.1011)

The RMSF values for the active site amino acid residues of 3CLpro before and after binding to Glabrene, are given in brackets as follows:

Thr24(0.1558,0.1515),Phe140(0.2036,0.0782),Asn142 (0.2433,0.1458),

Gly143(0.2714,0.1455), Cys 145(0.1389,0.0769), His163(0.1451,0.0689), His164(0.1433,0.0871), Glu166( 0.0812,0.0598),His172(0.0746,0.0727)

The RMSF values for the active site amino acid residues of RdRp before and after binding to Naringenin, are given in brackets as follows:

Cys306(0.0841,0.0840), Cys310(0.0972,0.0822), His 642(0.1429,0.0967), Cys645(0.1099,0.0863), Cys646(0.0916,0.0794).

For all the target proteins, higher RMSF values were observed for all active site amino acid residues in 


\begin{tabular}{|c|c|c|c|c|c|c|c|}
\hline \multicolumn{7}{|c|}{ Table 2a: Molecular Docking Analysis of Phytochemical Actives with ACE-2 Receptor. } \\
\hline & $\begin{array}{c}\text { Binding } \\
\text { Energy } \\
(\boldsymbol{\Delta g})\end{array}$ & $\mathbf{H}$ - bond & $\begin{array}{c}\text { Inhibition } \\
\text { Constant } \\
(\boldsymbol{\mu m})\end{array}$ & $\begin{array}{c}\text { Inter- } \\
\text { molecular } \\
\text { Energy }\end{array}$ & $\begin{array}{c}\text { VDW-H bond } \\
\text { desolva-tion } \\
\text { Energy }\end{array}$ & $\begin{array}{c}\text { Ligand } \\
\text { Efficiency }\end{array}$ & $\begin{array}{c}\text { Electro-static } \\
\text { energy }\end{array}$ \\
\hline Dichamanetin & -9.80 & 6 & 65.84 & -12.27 & -12.03 & -0.28 & -0.24 \\
\hline Hetidine & -9.40 & 4 & 129.18 & -9.95 & -8.28 & -0.36 & -1.67 \\
\hline 6-Deacetyl Nimbin & -7.70 & 2 & 2.28 & -9.62 & -9.35 & -0.21 & -0.27 \\
\hline Lycorine & -7.46 & 3 & 3.43 & -8.0 & -6.4 & -0.36 & -1.61 \\
\hline Epicatechin-gallate & -7.43 & 10 & 3.58 & -10.45 & -10.13 & -0.23 & -0.32 \\
\hline Phaitanthrin D & -7.24 & 3 & 4.91 & -7.24 & -7.15 & -0.33 & -0.09 \\
\hline Belladine & -7.01 & 3 & 7.24 & -9.21 & -8.49 & -0.3 & -0.7 \\
\hline Quercetin & -6.61 & 4 & 14.18 & -8.26 & -8.15 & -0.3 & -0.11 \\
\hline Scutellarein & -6.25 & 5 & 26.42 & -7.62 & -7.54 & -0.3 & -0.08 \\
\hline Baicalin & -5.30 & 8 & 130.97 & -8.04 & -8.48 & -0.17 & 0.44 \\
\hline
\end{tabular}

\begin{tabular}{|c|c|c|c|c|c|c|c|}
\hline \multicolumn{7}{|c|}{ Table 2b: Molecular docking analysis of phytochemical actives with 3CLpro. } \\
\hline & $\begin{array}{c}\text { Binding } \\
\text { energy } \\
(\boldsymbol{\Delta} \mathbf{G})\end{array}$ & $\mathbf{H - b o n d}$ & $\begin{array}{c}\text { Inhibition } \\
\mathbf{c o n s t a n t} \\
(\boldsymbol{\mu \mathbf { m } )}\end{array}$ & $\begin{array}{c}\text { Inter- } \\
\text { molecular } \\
\text { energy }\end{array}$ & $\begin{array}{c}\text { VDW-H bond } \\
\text { desolvation } \\
\text { Energy }\end{array}$ & $\begin{array}{c}\text { Ligand } \\
\text { efficiency }\end{array}$ & $\begin{array}{c}\text { Electro-static } \\
\text { energy }\end{array}$ \\
\hline Glabrene & -9.11 & 3 & 208.69 & -10.01 & -9.92 & -0.38 & -0.09 \\
\hline Luteolin & -8.54 & 5 & 546.58 & -9.92 & -9.74 & -0.41 & -0.17 \\
\hline Ferulic acid & -8.05 & 4 & 1.25 & -9.15 & -9.03 & -0.4 & -0.12 \\
\hline Epicatechin & -7.68 & 9 & 2.36 & -9.32 & -9.03 & -0.37 & -0.29 \\
\hline Apigenin & -7.59 & 6 & 2.72 & -8.69 & -8.65 & -0.38 & -0.04 \\
\hline Kaempferol & -7.42 & 8 & 3.66 & -8.79 & -8.51 & -0.35 & -0.27 \\
\hline Rosmarinic acid & -6.97 & 9 & 7.75 & -10.27 & -10.14 & -0.27 & -0.13 \\
\hline Caffeic acid & -4.99 & 6 & 219.47 & -6.36 & -6.3 & -0.38 & -0.07 \\
\hline Gallic acid & -4.33 & 6 & 666.2 & -5.71 & -5.6 & -0.36 & -0.11 \\
\hline Vanillic acid & -4.27 & & 746.1 & -5.36 & -5.54 & -0.36 & 0.17 \\
\hline
\end{tabular}

\begin{tabular}{|c|c|c|c|c|c|c|c|}
\hline Name & $\begin{array}{c}\text { Binding } \\
\text { energy } \\
(\Delta \mathrm{g})\end{array}$ & $\mathrm{H}$ - bond & $\begin{array}{c}\text { Inhibition } \\
\text { constant } \\
(\mu \mathrm{m})\end{array}$ & $\begin{array}{c}\text { Inter } \\
\text { molecular } \\
\text { energy }\end{array}$ & $\begin{array}{c}\text { VDW-H bond } \\
\text { desol-vation } \\
\text { Energy }\end{array}$ & $\begin{array}{l}\text { Ligand } \\
\text { efficiency }\end{array}$ & $\begin{array}{c}\text { Electro-static } \\
\text { energy }\end{array}$ \\
\hline Naringenin & -7.7 & 7 & 2.29 & -8.79 & -8.65 & -0.39 & -0.14 \\
\hline Licoflavonol & -7.00 & 6 & 7.45 & -8.92 & -8.74 & -0.27 & -0.18 \\
\hline Baicalein & -6.95 & 3 & 8 & -8.05 & -7.97 & -0.35 & -0.08 \\
\hline Vitexin & -6.69 & 9 & 12.54 & -9.43 & -8.9 & -0.22 & -0.53 \\
\hline LicocoumarinA & -6.49 & 3 & 17.54 & -8.68 & -8.56 & -0.22 & -0.12 \\
\hline Liquiritin & -6.22 & 6 & 27.36 & -8.69 & -7.91 & -0.21 & -0.78 \\
\hline Biochanin A & -6.08 & 6 & 34.74 & -7.18 & -6.67 & -0.29 & -0.51 \\
\hline Ellagic acid & -5.68 & 6 & 68.63 & -6.78 & -6.42 & -0.26 & -0.36 \\
\hline Licocoumarone & -5.44 & 5 & 103.31 & -7.36 & -7.36 & -0.22 & 0 \\
\hline Curcumin & -5.12 & 6 & 176.06 & -7.87 & -7.22 & -0.19 & -0.65 \\
\hline
\end{tabular}

free unbound state, making them more flexible and consequently unstable. RMSF values were lower when these residues were bound to respective phytochemical active ligands forming their complexes. Such complexes with lower RMSF values became less flexible, more rigid and more stable. This once again attests to their higher stabilities when compared to the free forms of their proteins.

\section{Multiple Sequence Alignment (MSA) of ACE-2}

Prevention of viral entry into host cells remains the first point of disease control. Since the Homo sapiens ACE-2 protein receptor is an entry point for binding with RBD of SARS-CoV-2 spike protein for cell invasion, MSA was used to find whether specific amino acid residues involved in this binding shows conservation in ACE-2 protein receptor of other species. The nine significant amino acid residues are $G \ln 24$, Asp38, Tyr41, Gln42, 


\begin{tabular}{|c|c|c|c|c|c|}
\hline $\begin{array}{l}\text { Phytochemical } \\
\text { ligands }\end{array}$ & $\begin{array}{l}\text { Hydrogen } \\
\text { bonds }\end{array}$ & $\begin{array}{l}\text { Hydrophobic } \\
\text { interactions }\end{array}$ & Salt bridges & $\pi$-stacking & $\begin{array}{c}\text { Cation- } \pi \\
\text { interactions }\end{array}$ \\
\hline Dichamanetin & 6 & 5 & 0 & 1 & 0 \\
\hline Hetidine & 4 & 3 & 1 & 0 & 0 \\
\hline 6-DeacetylNimbin & 2 & 3 & 2 & 0 & 0 \\
\hline Lycorine & 3 & 2 & 1 & 0 & 0 \\
\hline Epicatechin gallate & 10 & 2 & 0 & 1 & 1 \\
\hline Phaitanthrin D & 3 & 4 & 0 & 0 & 0 \\
\hline Belladine & 3 & 4 & 0 & 1 & 0 \\
\hline Quercetin & 4 & 6 & 0 & 0 & 0 \\
\hline Scutellarein & 5 & 6 & 0 & 0 & 0 \\
\hline Baicalin & 8 & 2 & 0 & 1 & 0 \\
\hline
\end{tabular}

\begin{tabular}{|c|c|c|c|c|c|}
\hline Phytochemical ligands & $\begin{array}{l}\text { Hydrogen } \\
\text { bonds }\end{array}$ & $\begin{array}{l}\text { Hydrophobic } \\
\text { interactions }\end{array}$ & Salt bridges & m-stacking & $\begin{array}{c}\pi \text {-cation } \\
\text { interactions }\end{array}$ \\
\hline Glabrene & 3 & 3 & 0 & 0 & 0 \\
\hline Luteolin & 5 & 2 & 0 & 0 & 0 \\
\hline Ferulic acid & 4 & 2 & 0 & 0 & 0 \\
\hline Epicatechin & 9 & 2 & 0 & 0 & 0 \\
\hline Apigenin & 6 & 2 & 0 & 1 & 0 \\
\hline Kaempferol & 8 & 2 & 0 & 0 & 0 \\
\hline Rosmarinic acid & 9 & 3 & 2 & 0 & 0 \\
\hline Caffeic acid & 6 & 3 & 0 & 0 & 0 \\
\hline Gallic acid & 6 & 4 & 0 & 0 & 0 \\
\hline Vanillic acid & 4 & 3 & 0 & 1 & 0 \\
\hline
\end{tabular}

\begin{tabular}{|c|c|c|c|c|c|}
\hline \multicolumn{2}{|c|}{ Table S3: Interactions of phytochemical actives with RdRp. } \\
\hline Phytochemical ligands & $\begin{array}{c}\text { Hydrogen } \\
\text { bonds }\end{array}$ & $\begin{array}{c}\text { Hydrophobic } \\
\text { interactions }\end{array}$ & Salt bridges & $\begin{array}{c}\pi \text {-stacking } \\
\text { interactions }\end{array}$ \\
\hline Naringenin & 7 & 9 & 0 & 0 & 0 \\
\hline Licoflavonol & 6 & 7 & 0 & 0 & 0 \\
\hline Baicalein & 3 & 10 & 0 & 0 & 0 \\
\hline Vitexin & 9 & 3 & 0 & 0 & 1 \\
\hline LicocoumarinA & 3 & 2 & 1 & 0 & 0 \\
\hline Liquiritin & 6 & 3 & 0 & 0 & 0 \\
\hline Biochanin A & 6 & 2 & 2 & 0 & 1 \\
\hline Ellagic acid & 9 & 1 & 0 & 0 & 0 \\
\hline Licocoumarone & 5 & 4 & 0 & 0 \\
\hline Curcumin & 6 & 4 & & 0 & 0 \\
\hline
\end{tabular}



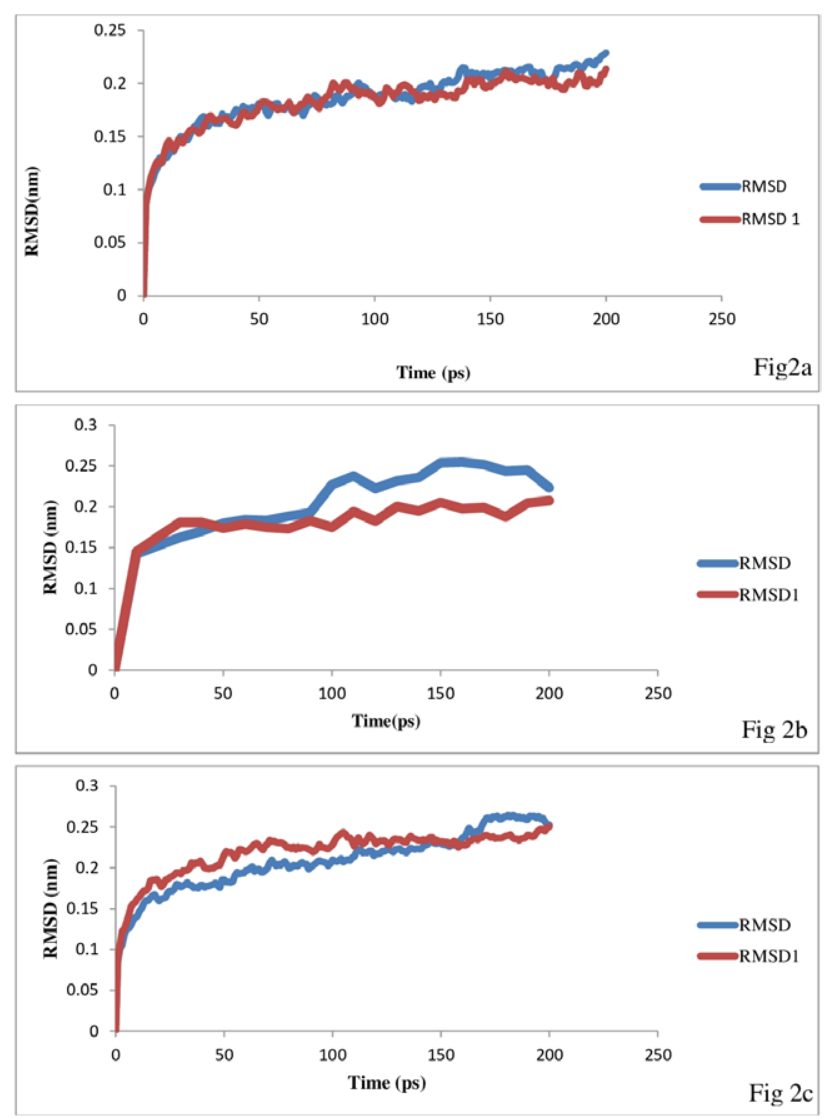

Figure 2: RMSD plot of free ACE-2 protein receptor (blue) and ACE-2- Dichamanetin complex (red), free 3CL Pro (blue) and 3CL Pro-Glabrene complex (red) and free RdRp (blue) and RdRp-Naringenin (red) complex.
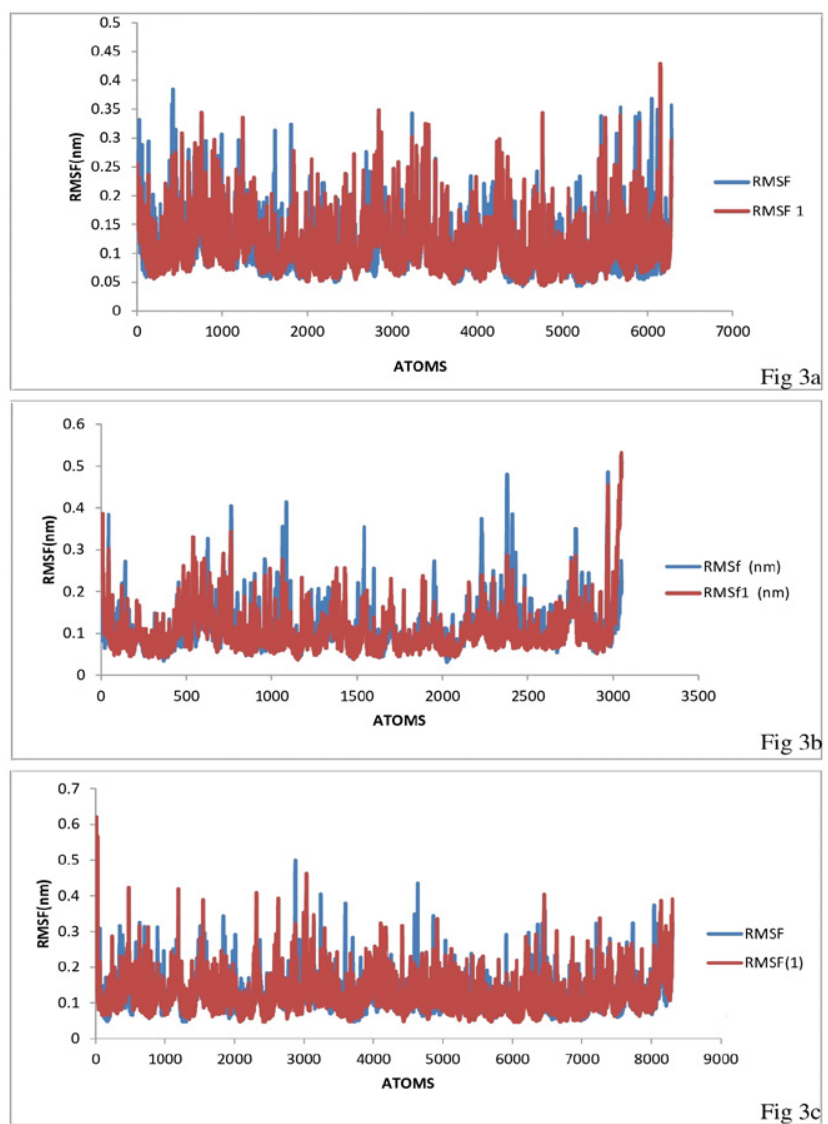

Figure 3: RMSF plot of free ACE-2 protein receptor (blue) and ACE-2- Dichamanetin complex (red), free 3CL Pro (blue) and 3CL Pro-Glabrene complex (red) and free RdRp (blue) and RdRp-Naringenin (red) complex.

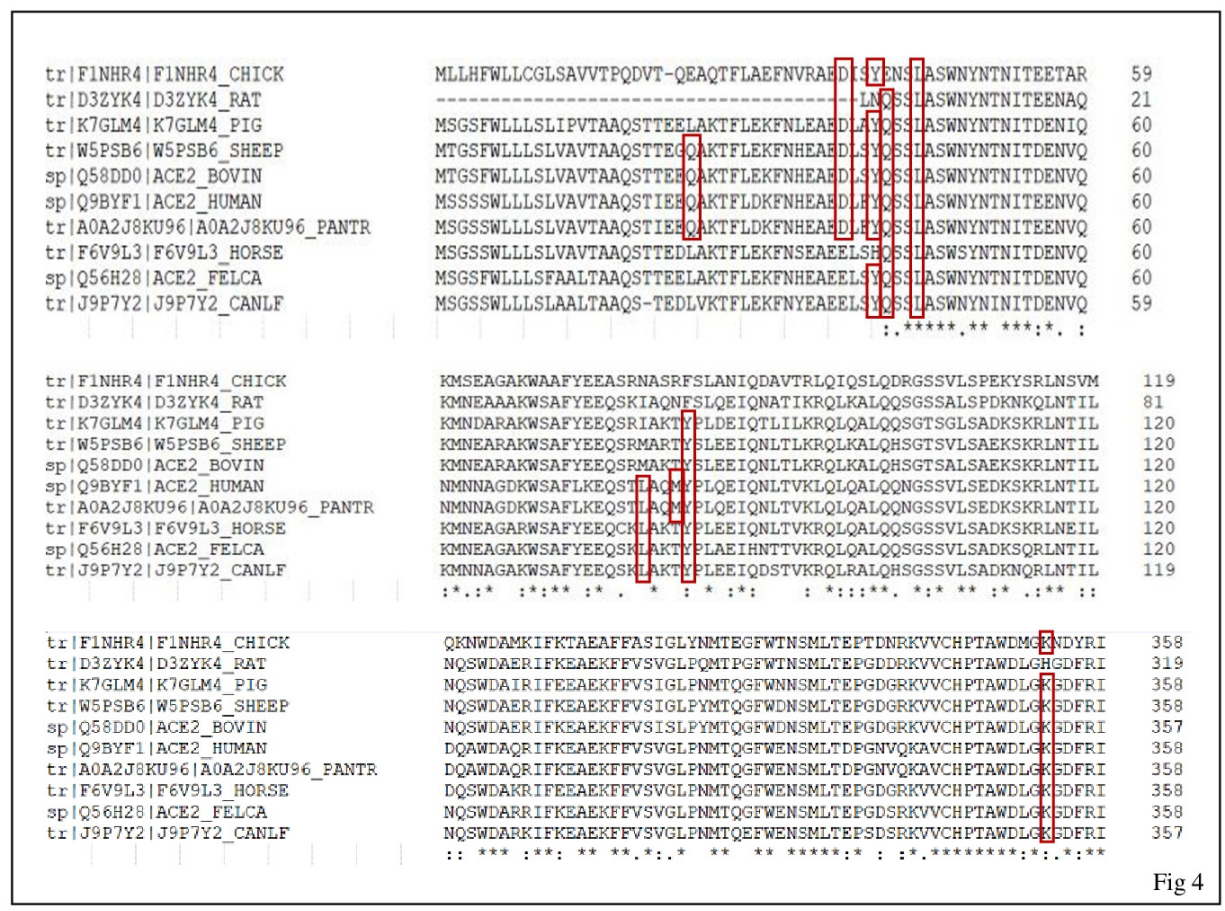

Figure 4: Multiple Sequence Alignment of Homo sapiens ACE-2 protein with different species, viz., Gallus galluss (chicken), Ratus norvegicus (Rat), Sus scrofa (Pig), Bos taurus (Bovine), Ovis aries (Sheep), Pan troglodytes (Chimpanzee), Equus caballus (horse), Canis lupus familiaris (Dog) and Felis catus (Cat). 
Table 3: Multiple sequence analysis of Homo sapien ACE-2 protein receptor amino acid residues with that of different species.

\begin{tabular}{|c|c|c|c|c|c|c|c|c|c|}
\hline \multirow{2}{*}{$\begin{array}{c}\text { Species Name (Common name I } \\
\text { conserved residues) }\end{array}$} & \multicolumn{7}{|c|}{ Amino Acid Residues } \\
\hline & Gln24 & Asp38 & Tyr41 & Gln42 & Leu45 & Leu79 & Met82 & Tyr83 & Lys353 \\
\hline Homo Sapiens (Human) & $\checkmark$ & $\checkmark$ & $\checkmark$ & $\checkmark$ & $\checkmark$ & $\checkmark$ & $\checkmark$ & $\checkmark$ & $\checkmark$ \\
\hline Pan troglodytes (Chimpanzee,9) & $\checkmark$ & $\checkmark$ & $\checkmark$ & $\checkmark$ & $\checkmark$ & $\checkmark$ & $\checkmark$ & $\checkmark$ & $\checkmark$ \\
\hline Bostaurus (Bovine,7) & $\checkmark$ & $\checkmark$ & $\checkmark$ & $\checkmark$ & $\checkmark$ & & & $\checkmark$ & $\checkmark$ \\
\hline Ovisaries (Sheep,7) & $\checkmark$ & $\checkmark$ & $\checkmark$ & $\checkmark$ & $\checkmark$ & & & $\checkmark$ & $\checkmark$ \\
\hline Canislupus familiaris (Dog,6) & & & $\checkmark$ & $\checkmark$ & $\checkmark$ & $\checkmark$ & & $\checkmark$ & $\checkmark$ \\
\hline Feliscatus (Cat,6) & & & $\checkmark$ & $\checkmark$ & $\checkmark$ & $\checkmark$ & & $\checkmark$ & $\checkmark$ \\
\hline Sus Scrofa (Pig,6) & & $\checkmark$ & $\checkmark$ & $\checkmark$ & $\checkmark$ & & & $\checkmark$ & $\checkmark$ \\
\hline Equuscaballus (Horse,5) & & & & $\checkmark$ & $\checkmark$ & $\checkmark$ & & $\checkmark$ & $\checkmark$ \\
\hline Gallus gallus (Chicken,4) & & $\checkmark$ & $\checkmark$ & & $\checkmark$ & & & & $\checkmark$ \\
\hline RatusNorvegicus (Rat,2) & & & & $\checkmark$ & $\checkmark$ & & & & \\
\hline
\end{tabular}

Leu45, Leu79, Met82, Tyr83 and Lys353. ${ }^{30}$ Their MSA withACE-2 protein receptors of nine species in Clustal Omega showed that these RBD specific amino acids are conserved in them to different extents (Figure 4, Table 3). Interestingly, $100 \%$ of residues are conserved in chimpanzees with $78 \%$ conservation (all except Leu79 and Met82) in livestock species like bovine and sheep. Six of the same residues are conserved in dogs and cats (Gln24, Asp38 and Met82 are not conserved), showing $67 \%$ similarity, which is same as in pigs, where Asp38 is conserved and Leu79 was not. Only five and four residues are conserved in horses (56\%) and chickens (44\%), with rats conserving only two residues, vir; Gln42 and Leu45. Potential interspecies transmission through ACE-2 protein receptor between Homo sapiens and Pan troglodytes (chimpanzees) may be possible as the RBD of the viral spike protein can fit well into such receptors of both species. Species jump between Homo sapiens and livestock animals may be less probable as also between dogs and cats, via this receptor pathway. Such prospects become negligible in equines, poultry and rodents. In this context, it comes critical to block the ACE-2 receptor protein as the first line of defence against SARS-CoV-2.

\section{CONCLUSION}

All thirty phytochemicals used in this study obeyed Lipinski's rule of five. The negative binding energies that accompany their molecular docking with respective target proteins, viz:, ACE-2 protein receptor, 3CLpro and RdRp, attested good complex stabilities. MDS studies, on target proteins and their model complexes with Dichamanetin, Glabreneand Naringenin showed RMSD and RMSF values favouring complex formation. All phytochemicals are available from plant sources. Hence their dietary therapeutic prospects, either as extracted and purified forms, or as direct plant products, can be further explored. Combinations of actives from each group (eg., Ashoka tree leaves, licorice/garden sage and oranges/tomato) may also have potential benefits for inhibition of viral entry and also targeting viral proteins. MSA of active residues in Homo sapiens ACE-2 protein receptor, with that of different species, revealed that residues are conserved to different extents in the species and hence possibilities of inter-species transmission of SARS-CoV-2 occurring by this receptor pathway exist among them.

\section{ACKNOWLEDGEMENT}

The authors are grateful to Vellore Institute of Technology, Vellore for supporting this project.

\section{CONFLICT OF INTEREST}

The authors declare no conflict of interest.

\section{ABBREVIATIONS}

ACE-2: Angiotensin-Converting Enzyme; 3CLpro: 3C Like Protease; RdRp: RNA dependent RNA polymerase; RBD: Receptor Binding Domain; MDS: Molecular Dynamic Simulation; RMSD:Root Mean Square Deviation; RMSF:Root Mean Square Fluctuation; MSA: Multiple Sequence Alignment.

\section{REFERENCES}

1. Chavez S, Long B, Koyfman A, Liang SY. Coronavirus Disease (COVID-19): A primer for emergency physicians. Am J Emerg Med. 2020;S07356757(20)30178-9 
2. Shereen MA, Khan S, Kazmi A, Bashir N, Siddique R. COVID-19 infection: Origin, transmission and characteristics of human coronaviruses. J Adv Res. 2020;24:91-8.

3. Huang C, Wang Y, Li X, Ren L, Zhao J, Hu Y, et al. Clinical features of patients infected with 2019 novel coronavirus in Wuhan, China. Lancet. 2020;395(10223):497-506.

4. Letko M, Marzi A, Munster V. Functional assessment of cell entry and receptor usage for SARS-CoV-2 and other lineage B betacoronaviruses. Nat Microbiol. 2020;5(4):562-9.

5. Hussain M, Jabeen N, Raza F, Shabbir S, Baig AA, Amanullah A, et al. Structural variations in human ACE2 may influence its binding with SARSCoV-2 spike protein. J Med Virol. 2020;92(9):1580-6.

6. Ortega JT, Serrano ML, Pujol FH, Rangel HR, Biology S. Role of changes in SARS-CoV2 spike protein in the interaction with the human ACE-2 receptor: An in silico analysis. EXCLI J. 2020;19:410-7.

7. Zhang L, Lin D, Sun X, Curth U, Drosten C, Sauerhering L, et al. Crystal structure of SARS-CoV-2 main protease provides a basis for design of improved a-ketoamide inhibitors. Science. 2020;368(6489):409-12.

8. Yang H, Xie W, Xue X, Yang K, Ma J, Liang W, et al. Design of wide-spectrum inhibitors targeting coronavirus main proteases. PLoS Biol. 2005;3(10):e324.

9. Pillaiyar T, Manickam M, Namasivayam V, Hayashi Y, Jung SH. An overview of severe acute respiratory syndrome-coronavirus (SARS-CoV) 3CL protease inhibitors: Peptidomimetics and small molecule chemotherapy. J Med Chem. 2016;59(14):6595-628.

10. Subissi L, Posthuma CC, Collet A, Zevenhoven-Dobbe JC, Gorbalenya AE, Decroly $\mathrm{E}$, et al. One severe acute respiratory syndrome coronavirus protein complex integrates processive RNA polymerase and exonuclease activities. Proc Natl Acad Sci USA. 2014;111(37):E3900-9.

11. Kirchdoerfer RN, Ward AB. Structure of the SARS-CoV nsp12 polymerase bound to nsp7 and nsp8 co-factors. Nat Commun. 2019;10(1):1-9.

12. Sanchis-Gomar F, Lavie CJ, Perez-Quilis C, Henry BM, Lippi G. Angiotensinconverting Enzyme 2 and antihypertensives (angiotensin receptor blockers and angiotensin-converting enzyme inhibitors) in coronavirus Disease 2019. Mayo Clin Proc. 2020;95(6):1222-30. https://doi.org/10.1016/j. mayocp.2020.03.026.

13. Anand K, Ziebuhr J, Wadhwani P, Mesters JR, Hilgenfeld R. Coronavirus main proteinase (3CLpro) Structure: Basis for design of anti-SARS drugs. Science. 2003;300(5626):1763-7.

14. Wang M, Cao R, Zhang L, Yang X, Liu J, Xu M, et al. Remdesivir and chloroquine effectively inhibit the recently emerged novel coronavirus (2019nCoV) in vitro. Cell Res. 2020;30(3):269-71.
15. Towler P, Staker B, Prasad SG, Menon S, Tang J, Parsons T, et al. ACE2 $\mathrm{X}$-Ray structures reveal a large hinge-bending motion important for inhibitor binding and catalysis. J Biol Chem. 2004;279(17):17996-8007.

16. Jin Z, Du X, Xu Y, Deng Y, Liu M, Zhao Y, et al. Structure of Mpro from COVID-19 virus and discovery of its inhibitors. Nature. 2020;582:289-93.

17. Gao Y, Yan L, Huang Y, Liu F, Zhao Y, Cao L, et al. Structure of the RNA-dependent RNA polymerase from COVID-19 virus. Science. 2020;7498(4):eabb7498.

18. O'Boyle NM, Banck M, James CA, Morley C, Vandermeersch T, Hutchison GR. Open Babel: An open chemical toolbox. J Cheminform. 2011;3(1):33.

19. Daina A, Michielin O, Zoete V. Swiss ADME: A free web tool to evaluate pharmacokinetics, drug-likeness and medicinal chemistry friendliness of small molecules. Sci Rep. 2017;7(1):42717.

20. Forli S, Huey R, Pique ME, Sanner MF, Goodsell DS, Olson AJ. Computational protein-ligand docking and virtual drug screening with the Auto Dock suite. Nat Protoc. 2016;11(5):905-19.

21. Salentin S, Schreiber S, Haupt VJ, Adasme MF, Schroeder M. PLIP: Fully automated

22. Protein-ligand interaction profiler. Nucleic Acids Res. 2015;43(W1):W443-7.

23. Lindahl E, Hess B, DerSpoel DV. GROMACS 3.0: A package for molecular simulation and trajectory analysis. J Mol Model. 2001;7(8):306-17.

24. Oostenbrink C, Villa A, Mark AE, Gunsteren WFV. A biomolecular force field based on the free enthalpy of hydration and solvation: The GROMOS forcefield parameter sets 53A5 and 53A6. J Comput Chem. 2004;25(13):1656-76

25. Schüttelkopf AW, Aalten DMFV. PRODRG: A tool for high-throughput crystallography of protein-ligand complexes. Acta Crystallogr Sect D Biol Crystallogr. 2004;60(8):1355-63.

26. Ragunathan A, Malathi K, Ramaiah S, Anbarasu A. FtsA as a cidal target for Staphylococcus aureus: Molecular docking and dynamics studies. J Cell Biochem. 2019;120(5):7751-8.

27. Sievers F, Wilm A, Dineen D, Gibson TJ, Karplus K, Li W, et al. Fast, scalable generation of high-quality protein multiple sequence alignments using Clustal Omega. Mol Syst Biol. 2011;7(1):539.

28. Andersen KG, Rambaut A, Lipkin WI, Holmes EC, Garry RF. The proximal origin of SARS-CoV-2. Nat Med. 2020;26(4):450-2.

29. Wu C, Liu Y, Yang Y, Zhang P, Zhong W, Wang Y, et al. Analysis of therapeutic targets for SARS-CoV-2 and discovery of potential drugs by computational methods. Acta Pharm Sin B. 2020;10(5):766-88.

30. Pollastri MP. Overview on the rule of five. Curr Protoc Pharmacol 2010; (Suppl. 49):1-8.

31. Lan J, Ge J, Yu J, Shan S, Zhou H, Fan S, et al. Structure of the SARSCoV-2 Spike receptor-binding domain bound to the ACE2 receptor. Nature. 2020;581(7807):215-20.

\section{SUMMARY}

COVID-19 is a globally raging pandemic disease. Drug development strategies for its treatment can either include using actives to block the angiotensin-converting enzyme-2 (ACE-2) protein receptor of host cells and prevent binding of the causative SARS-CoV-2 to this entry point, or inactivate essential viral proteases inside these cells. Accordingly, in this present study, three sets of ten phytochemical actives were virtually explored as therapeutic options for the same. Viral proteins studied for binding with actives were 3CLpro and RNA dependent RNA polymerase (RdRp). All the phytochemicals obeyed Lipinski's rule of five and showed favourable binding energies with the respective target proteins, attesting the stability of complexes. Among each set of actives, Dichamanetin-ACE-2, Glabrene-3CLpro and Naringenin-RdRp complexes were most stable, with binding energies of $-9.8,-9.11$ and $-7.7 \mathrm{Kcal} / \mathrm{mol}$ respectively. Molecular dynamic simulation studies, carried out for these representative actives and their complexes, also attested complex stabilities. Possibly these actives can be consumed, in combination as a dietary therapy for blocking viral entry into host cells and for binding to 3CLpro and RdRp for their inactivation. In addition multiple sequence alignment of the nine significant amino acid residues of the Homo sapiens ACE-2 protein receptor that interact with the receptor-binding domain of SARSCoV-2, with those of nine different species, was performed. All residues of the Homo sapiens ACE-2 protein receptor were conserved in Pan troglodytes (chimpanzee). The results reveal the possibility of interspecies transmission through the ACE-2 protein receptor and the importance of blocking it by therapeutic options. 


\section{PICTORIAL ABSTRACT}
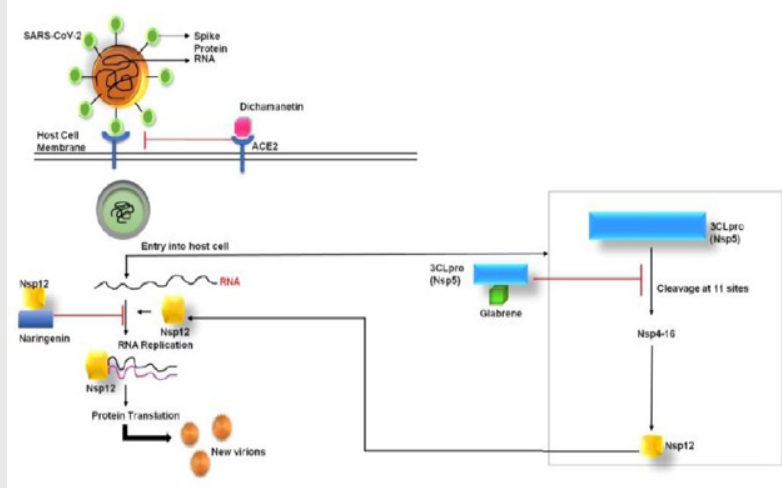

\section{About Authors}

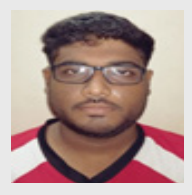

Dipjyoti Dey, M.Sc Applied Microbiology, School of Biosciences and Technology, Vellore Institute of Technology, Vellore, India. Area of Research \& Domain of Research: Bioinformatics and Drugs Designing.

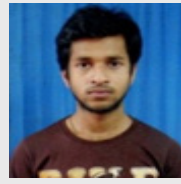

Nirban Dey, M.Sc Applied Microbiology, School of Biosciences and Technology, Vellore Institute of Technology, Vellore, India. Area of Research \& Domain of Research: Bioinformatics, Infectious disease biology.

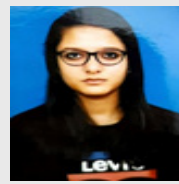

Shalini Ghosh, M.Sc Applied Microbiology, School of Biosciences and Technology, Vellore Institute of Technology, Vellore, India. Area of Research \& Domain of Research: Bioinformatics, Medical Microbiology.

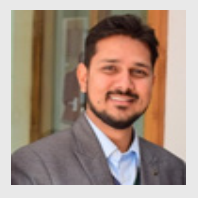

Dr. Imran Khan (Ph.D), Postdoctoral Scientist, Department of Molecular Biology, Bezmialem Vakif University, Istanbul, Turkey. Area of Research \& Domain of Research: Translational research for glioma and brain metastasis investigating mechanisms of brain tumors, establishing new effective treatments and finding new diagnostic and prognostic biomarkers.

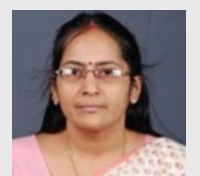

Dr. Padma Thiagarajan (Ph.D), Professor (Higher Academic Grade), School of Biosciences and Technology, Vellore Institute of Technology, Vellore, India. Area of Research \& Domain of Research: Biomaterials and Applied Biotechnology, Bioinformatics.

Cite this article: Dey D, Dey N, Ghosh S, Khan I, Thiagarajan P. Therapeutic Phytochemical Actives for Potential Control of SARS-CoV-2. Indian J of Pharmaceutical Education and Research. 2021;55(2):517-26. 